\title{
Codon optimization to PCR
}

\section{Recent introductions include an array of heart disease genes.}

$A^{2}$ reader

Beckman Coulter www.beckmancoulter.com Fluorescence-based CCD microplate reader

The $A^{2}$ is designed for automatic quantification of hundreds of protein analytes on the $A^{2}$ microarray plate as part of Beckman Coulter's $A^{2}$ Microarray System for multiplexed immunoassays. The $A^{2}$ system allows users to detect and quantify protein-protein interactions, protein immunoassays, DNA-protein interactions, enzyme reactions and other

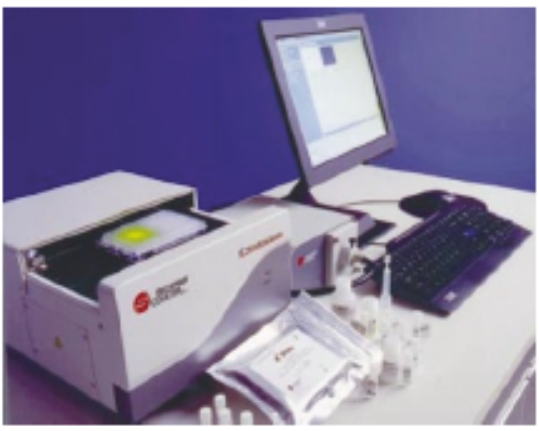

A square deal: the $\mathbf{A}^{2}$ microplate reader.

protein assays. The reader, about the size of a laptop computer, can read a plate and process microarray data in under five minutes. Built-in software aligns a grid around each of the spots on the microarray plates and measures the fluorescent inten sity. The $\mathrm{A}^{2}$ reader can also beintegrated with Biomekautomated liquid-handling platforms.

Cardiovascular disease gene array II

Superarray Bioscience www.superarray.com Vital information

This new product in the GEArray Q Series is designed to analyse the relative expression of 96 atherosclerosis-related genes. The human genes on thisarrayare important for the onset, progression and prevention of atherosclerosis. Through simple side-by-side hybridization, investigators can simultaneously determine the expression profile of these endothelial cell genes using experimental RNA samples, the arrays provided in the kit, and a SuperArray Probe Synthesis kit. An array containing the equivalent mousegenes willalso be available.

\section{Advalytix ArrayBooster}

GRI Molecular Biology www.gri.co.uk Agitation beats diffusion

Most microarray substrates are conventional microscope slides with up to several thousand spots of oligonucleotides or cDNA probes with known identity covering the slide in a checkerboard pattern. The sample is usually sandwiched between the DNAmicroarrayand a cover slip, forming a reaction chamber, and diffusion is the only mechanism by which the DNA strands move within the capillary gap. The Advalytix ArrayBooster uses agitation based on surface acoustic waves, rather than diffusion, to mix the sample during incubation. This allows the system to reach equilibrium in an overnight hybridization, increases the signal-to-noise ratio, improves slide-to-slide reproducibility and enhances homogeneityacrossthearray.

\section{Platinum PCR SuperMix High Fidelity}

Invitrogen www.invitrogen.com Double advantage

The Platinum PCR SuperMix High Fidelity is described as combining the robustness, fidelity and yields of Platinum Taq DNA Polymerase High Fidelity with the time saving, simplicity and high productivity of a PCR supermix. The enzyme increases PCR fidelity approximately sixfold over Taqpolymerase alone. Theformat is pre-mixed and needs only to be added to template and primers to begin PCR.

Codon optimization service

GenScript

www.genscript.com

A boost for protein expression

Codon preferences can vary widely between species. To enhance the expression level of a foreign protein in a given system $(E$, coli, yeast, insect or mammalian cell), it is important to adjust the codon frequency of the foreign protein to match the host expression system. An algorithm developed by GenScript optimizes sequences for protein expression based on the user's codon table, or on a publicly available codon usage database. The algorithm converts an amino acid sequence into a DNA sequence with overall codon usage similar to a specified organism, and optimizes RNA secondary structure, GC content and repetitive codons.

Low-volume UVmicroplates

Corning Life Sciences www.corning.com Do it by halves

Corning's half-area, 96-well UV microplate for quantification of DNA, RNA and proteins features a novel well height that creates a samplevolume of $175 \mathrm{ml}$. This corresponds directly with a $1 \mathrm{~cm}$ path length for calibration using UV absorbance, at the same time offering reductions inreagentcostsand the use oflimited samples. Corning claims that researchers will save significant amounts of time by using UV microplates rather than quartz cuvettes.
Thedisposable microplates eliminatewashing andrecycling, andalso removetherisk of damaging fragile and expensive quartz cuvettes. A single half-area microplate is sufficient for all steps, fromcalibration todirect measurements of nucleotide or protein concentrations. The plates are made of high-transmittance polymer that has low background for consistent detection of samples at $260 \mathrm{~nm}$ and $280 \mathrm{~nm}$. The UV-transparent bottom is moulded directly onto the plate, creatingan integral seal that prevents leaks and cross-contamination.

These notes are compiled in the Nature office from information provided bythemanufacturers. 